\title{
Cytokine-induced killer cells carrying recombinant oncolytic adenovirus expressing P21Ras scFv inhibited liver cancer
}

\author{
Fang Dai1 ${ }^{1, \#}$, Peng-Bo Zhang ${ }^{3 \#, ~ Q i a n g ~ F e n g ~}{ }^{2}$, Xin-Yan Pan², Shu-Ling Song ${ }^{2}$, Jing Cui ${ }^{2}$ and Ju-Lun Yang ${ }^{1,2}$ \\ 1. Graduate School, Kunming Medical University, Kunming, Yunnan, China. \\ 2. $920^{\text {th }}$ Hospital of the Joint Logistics Support Force of PLA, Kunming, Yunnan, China. \\ 3. Medical School, Kunming University of Science and Technology, Kunming, Yunnan, China. \\ \#Co-first authors.
}

$\square$ Corresponding author: Ju-Lun Yang, MD, PhD, Department of Pathology, 920th Hospital of the Joint Logistics Support Force of PLA, 212 Daguan Road, Kunming, Yunnan, China. Tel: (+86) 64774769; Fax: (+86) 64774769. E-mail: daifang@kmmu.edu.cn.

(C) The author(s). This is an open access article distributed under the terms of the Creative Commons Attribution License (https://creativecommons.org/licenses/by/4.0/). See http://ivyspring.com/terms for full terms and conditions.

Received: 2020.08.03; Accepted: 2021.02.18; Published: 2021.03.10

\begin{abstract}
Background: Oncolytic adenovirus-mediated gene therapy is an emerging strategy for cancer treatment. However, oncolytic adenoviruses are mainly administered locally at tumor site. Intravenous administration of oncolytic adenovirus for cancer gene therapy is a problem that needs to be solved urgently.

Methods: We constructed recombinant oncolytic adenovirus KGHV500 carrying anti-p21Ras scFv and employed CIK cells to deliver KGHV500. TUNEL, wound healing, MTT, and Transwell invasion assays were used to determine the anti-tumor efficacy of KGHV500 on liver cancer cells in vitro. Nude mouse xenograft model was used to examine the anti-tumor efficacy of CIK cells combined with KGHV500 in vivo. Furthermore, KGHV500 accumulation in different organs was detected to assess the safety.

Results: KGHV500 inhibited the migration, proliferation, invasion, and induced the apoptosis of liver cancer cells. CIK cells carrying KGHV500 reached tumor site and exerted much better anti-tumor efficacy than CIK cells or KGHV500 alone in nude mouse xenograft model. Moreover, we detected KGHV500 and anti-p21Ras scFv in different organs of nude mice, with little effects on the organs.

Conclusions: We develop a novel strategy for the treatment of Ras-driven liver cancer by combining CIK cells with oncolytic adenovirus expressing anti-p21Ras scFv. Intravenous injection of CIK cells carrying KGHV500 in vivo significantly inhibits tumor growth, has little effect on normal organs, and is relatively safe.
\end{abstract}

Key words: oncolytic adenovirus, Ras, liver cancer, $\mathrm{CIK}$, scFv

\section{Introduction}

Liver cancer is a common malignant tumor worldwide, with the fourth highest mortality rate [1]. In China, the incidence of liver cancer is high, and liver cancer has the third highest mortality rate [2]. The current treatment options for liver cancer include surgical resection, local ablation, liver transplantation, radiation therapy, transcatheter arterial chemoembolization, and systemic chemotherapy, but the 5-year survival rate of liver cancer patients remains extremely low [3]. While multi-target kinase inhibitor sorafenib could prolong median survival of patients with advanced liver cancer, approximately $70 \%$ of patients develop resistance or serious adverse reactions to sorafenib [4-9].

Ras is a classic oncogene involved in cell proliferation, differentiation, and apoptosis [10]. Ras mutations contribute to $30 \%$ of all human cancers [11]. Among three Ras genes KRAS, NRAS, and HRAS, NRAS and HRAS mutations are more common in liver cancer [12]. Furthermore, HRAS overexpression 
plays a significant role in the development of liver cancer [13]. Therefore, Ras is a potential target for liver cancer therapy.

In previous study, we constructed p21-Ras single-chain variable fragment antibody (anti-p21Ras $\mathrm{scFv}$ ) that could bind mutant p21Ras and overexpressed p21Ras (K-p21Ras, N-p21Ras, and H-p21Ras) [14]. In addition, we utilized recombinant oncolytic adenovirus KGHV300 carrying anti-p21Ras $\mathrm{scFv}$ for intratumoral injection to inhibit tumor growth in vivo [15]. However, intratumoral injection is only suitable for superficial tumors and is not useful for deep tumors. Intravenous injection of adenovirus in vivo therapy has the hidden danger of systemic virus infection [16]. Therefore, it is particularly urgent to solve the problem of adenovirus delivery from veins to tumors. Cytokine-induced killer (CIK) cells can recognize tumors through related receptors and will not damage normal cells. In present study, we employed CIK cells to deliver KGHV500 into the tumor and investigated anti-tumor effects and safety in vivo.

\section{Materials and Methods}

\section{Cell lines}

Human liver cancer Huh7 cell line, normal liver LO-2 cell line, and HEK293 cells were obtained from Kunming Institute of Zoology, Chinese Academy of Sciences. Cells were cultured in DMEM supplemented with $10 \%$ heat-inactivated fetal bovine serum (FBS), $100 \mathrm{U} / \mathrm{mL}$ penicillin and $100 \mu \mathrm{g} / \mathrm{mL}$ streptomycin at $37^{\circ} \mathrm{C}$ with $5 \% \mathrm{CO}_{2}$ atmosphere.

\section{Recombinant oncolytic adenovirus construction}

Wild-type adenovirus (Ad5) was used to construct recombinant oncolytic adenoviruses KGHV400 and KGHV500. Ad5 cilia gene was replaced with Ad35 cilia gene to promote virus binding to CD46 receptor on CIK cells. In KGHV500, anti-p21Ras scFv gene was inserted to express antip21Ras scFv constitutively, while in KGHV400 there was no insert of anti-p21Ras scFv gene. HEK293 cells were used to amplify KGHV400 and KGHV500. Virus stocks were collected, and purified by $\mathrm{CsCl}$ density gradient centrifugation. Virus titer was determined by the TCID50 method. Huh7 and CIK cells were infected with viruses at a MOI of 100 .

\section{Electron microscopy}

The cells were fixed in $2 \%$ glutaraldehyde solution for $40 \mathrm{~min}$, washed with PBS and fixed with $1 \%$ osmium acid for $30 \mathrm{~min}$. Next the samples were dehydrated with ethanol and embedded in EPON 618 epoxy resin. The embedded samples were cut into 1-2 $\mu \mathrm{m}$ sections using an ultramicrotome, stained with uranyl acetate, and observed under electron microscope.

\section{Preparation of CIK cells}

Peripheral blood mononuclear cells (PBMCs) were isolated from healthy volunteers by lymphocyte separation and density gradient centrifugation. PBMCs were cultured in RPMI 1640 medium containing $10 \% \quad$ FBS and $1,000 \quad \mathrm{U} / \mathrm{mL}$ IFN- $\gamma$ (Peprotech, USA). The medium was replaced every three days, and $300 \mathrm{U} / \mathrm{mL}$ IL-2 was added. After 14 days, CIK cells were harvested at $90 \%$ confluency. CIK phenotypes were characterized by immunohistochemistry. Briefly, the cells were incubated with anti-CD3 mAb (ZSGB-Bio, ZA0503, China), anti-CD56 mAb (MXB, Kit-0028, China), and anti-CD46 mAb (Abcam, EPR4014, UK) overnight at 4 ${ }^{\circ} \mathrm{C}$. After washing, the cells were incubated with horseradish peroxidase labeled goat anti-mouse IgG antibody (ZSGB-Bio, ZB5305, China) at $37{ }^{\circ} \mathrm{C}$ for 30 min, and then incubated with diaminobenzidine. Counterstaining was performed using hematoxylin, and the slides were sealed with neutral balsam.

\section{Flow cytometry}

CIK cells were incubated with anti-CD16-APC (eBioscience), anti-CD56-PE (Biolegend), and antiCD3-PerCP (Biolegend). Samples were centrifuged for $10 \mathrm{~min}$ at 1,500 rpm at room temperature, washed twice in PBS and subjected to flow cytometry on FC500 cytometer (Beckman-Coulter). The results were analyzed using Flow Jo software.

\section{MTT assay}

Huh7 cells were plated at a density of $1 \times 10^{4}$ cells per well in 96-well plates. After 1-3 d, cells were infected with KGHV500 at a MOI of 100. Then, $20 \mu \mathrm{L}$ MTT agent (Amresco, USA) was added to each well on $1,2,3,4$, and 5 days after infection. After incubation for $4 \mathrm{~h}$ at $37{ }^{\circ} \mathrm{C}$, MTT solution was removed and $100 \mu \mathrm{L}$ dimethyl sulfoxide was added to each well, and the plates were shaken for $10 \mathrm{~min}$. The optical density (OD) value of each well was measured in a microplate reader (Bio-Rad).

\section{Wound healing assay}

Huh7 cells were infected with KGHV500 at a MOI of 100. After $24 \mathrm{~h}$, the cells were seeded in 6-well plates and cultured to $90 \%$ confluency. A straight scratch was made in the cell monolayer with a $200 \mu \mathrm{L}$ tip, and the cells were washed three times with PBS to remove detached cells. Cells were cultured for $48 \mathrm{~h}$, and cell migration over the scratch was monitored with an inverted microscope. The migration distance was measured with ImageJ software. 


\section{Transwell invasion assay}

Transwell insert (Corning 3422 USA) was divided into upper and lower chambers by a microporous membrane. The upper chamber was coated with Matrigel. Huh7 cells were infected with KGHV500 at a MOI of 100 . After $24 \mathrm{~h}$, the cells were seeded in the upper chamber, and $500 \mu \mathrm{L}$ of medium was added to the lower chamber. Cells were incubated in the Transwell insert for $24-48 \mathrm{~h}$ at $37^{\circ} \mathrm{C}$. The cells in the upper chamber were removed with cotton swab, and the membranes were fixed in methanol for 15 min and stained with Giemsa for 15 min. Cells that migrated through the Matrigel were counted under an inverted microscope, and five fields in each sample were counted.

\section{TUNEL assay}

Cells or tumor sections were fixed and stained using TUNEL kit (Roche Diagnostics, Indianapolis, IN, USA) following the manufacturer's protocols. The nuclei were stained as blue by 4',6-diamidino-2phenylindole.

\section{Animal xenograft model}

Huh7 $\left(1 \times 10^{7} / 200 \mu \mathrm{L}\right)$ cells were injected subcutaneously into the right axilla of BALB/C nude mice (Vital River Laboratories, Beijing, China). When the tumors grew to average diameter of $5 \mathrm{~mm}$, the animals were randomized into 5 groups and received intravenous injection of either PBS, CIK cells, KGHV500, CIK + KGHV500, or CIK + KGHV400, respectively. Tumor size was measured with Vernier caliper every three days. Tumor volume was calculated according to the formula: $V=$ (length $\times$ width $\left.^{2}\right) \div 2$ [16]. When tumors in PBS group became ulcerated, mice were sacrificed. The dissected tumors were embedded with paraffin for immunohistochemical analysis. The anti-FLAG tag antibody (Abnova, MAB9744, China) and anti-human adenovirus hexon mouse mAb (Novus, NBP211638, USA) were used to detect KGHV500 adenovirus.

\section{Real-time PCR}

Total RNA was extracted from tumor tissues using the ReliaPrep ${ }^{\mathrm{TM}}$ Total RNA extraction kit (Promega, USA). RNA was reverse transcribed into cDNA using the GoScript ${ }^{\mathrm{TM}}$ Reverse Transcription system (Promega, USA). PCR was performed with GoTaq qPCR Master Mix (Promega, USA). The primers were as follows: survivin CCACTGAGAACG AGCCAGAC and CGCACTTTCTCCGCAGTTTC; p53 CCTGAGGTTGGCTCTGACTG and CTTCTTT GGCTGGGGAGAGG; caspase-7 AAGCTGACTTCC TCTTCGCC and TCCAGGTCTTTTCCGTGCTC; caspase-3 AAATACCAGTGGAGGCCGAC and AAC
CCGGGTAAGAATGTGCA; bcl-2 GGGGTCATGTG TGTGGAGAG and ACCTACCCAGCCTCCGTTAT; GAPDH TGACAACAGCCTCAAGAT and GAGTC CTTCCACGATACC. The reaction conditions were as follows: $94^{\circ} \mathrm{C}$ for $15 \mathrm{~s}, 60^{\circ} \mathrm{C}$ for $30 \mathrm{~s}$, and $72{ }^{\circ} \mathrm{C}$ for $30 \mathrm{~s}$ (40 cycles). The data were analyzed using Bio-Rad CFX96 Manager software.

\section{Western blotting}

Mouse tissues were lysed in RIPA buffer on the ice for $30 \mathrm{~min}$. The lysates were centrifuged and the supernatants were collected for SDS-PAGE gel electrophoresis. The proteins were then transferred to polyvinylidene fluoride membranes, which were incubated with antibodies for survivin, p53, Caspase-7, Caspase-3, Bcl-2, and $\beta$-actin (all from Abcam) overnight. Next, the membranes were incubated with horseradish peroxidase labeled goat anti-mouse IgG antibody (ZSGB-BIO, Beijing, China), and developed with enhanced chemiluminescence kit (Pierce).

\section{Statistical analysis}

All data were expressed as mean \pm standard deviation and analyzed using SPSS 22.0 software (SPSS, Chicago, USA). Differences in multiple groups were analyzed by one-way analysis of variance (ANOVA). Statistical significance was indicated by P-value $<0.05$.

\section{Results}

\section{Infection of Huh7 cells with recombinant oncolytic adenovirus KGHV500}

Ras proteins $\mathrm{p} 21 \mathrm{~K}-$ Ras, $\mathrm{p} 21 \mathrm{H}-\mathrm{Ras}$, and $\mathrm{p} 21 \mathrm{~N}-$ Ras were overexpressed in Huh7 cells but not in normal LO-2 hepatocytes (Figure 1A). Huh7 and KGHV500 were co-cultured, and the optimal ratio of virus-infected cells was determined by monitoring the cytopathic effect (CPE). After 48-h infection, at a MOI of 100, CPE was weak, and fluorescence increased (Figure 1B). In addition, numerous virus particles were present in the cytoplasm and nuclei of Huh7 cells infected with oncolytic adenovirus (Figure 1C). CD46 was expressed on Huh7 cell membrane and bound to receptor of KGHV500 (Figure 1D). These results validated that Huh7 cells were successfully infected with KGHV500.

\section{KGHV500 inhibited liver cancer cell migration}

The wound healing assay demonstrated that the number of migrated cells in KGHV500-treated group was less than that of control group (Figure 2A). The percentage of migrated cells was $18.68 \pm 2.11 \%$ at $24 \mathrm{~h}$ and $47.14 \pm 3.86 \%$ at $48 \mathrm{~h}$ after KGHV500 infection; the percentage of migrated cells was $44.12 \pm 2.29 \%$ at $24 \mathrm{~h}$ 
and $68.29 \pm 3.70 \%$ at $48 \mathrm{~h}$ after KGHV400 infection. In Huh7 control group, the percentage of migrated cells was $49.55 \pm 3.10 \%$ at $24 \mathrm{~h}$ and $78.49 \pm 4.23 \%$ at $48 \mathrm{~h}$ (Figure 2B).

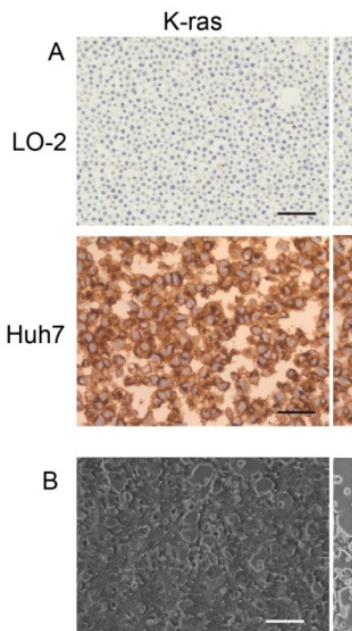

$\mathrm{MOI}=50$
$\mathrm{H}$-ras

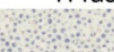

$\mathrm{N}$-ras
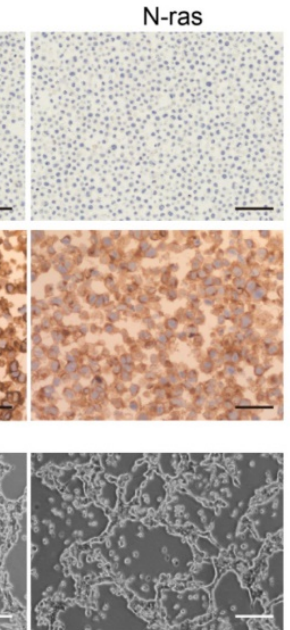

$\mathrm{MOI}=150$
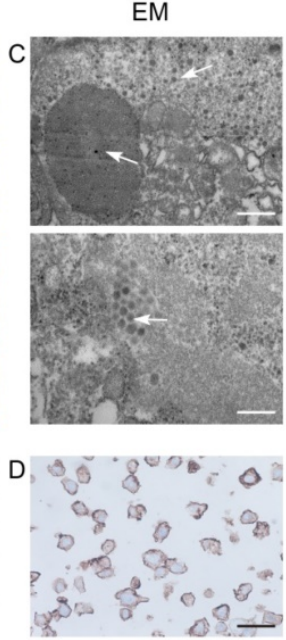

$\mathrm{CD} 46$

Figure 1. Characterization of KGHV500-infected Huh7 cells. (A) K-Ras, H-Ras, and N-Ras were stained strong in Huh7 liver cancer cells but not in LO-2 normal liver cells. Bar: $100 \mu \mathrm{m}$. (B) After $48 \mathrm{~h}$ of infection with KGHV500 (MOI = 100), the cytopathic effect (CPE) was the weakest. Bar: $200 \mu \mathrm{m}$. (C) Virus particles were observed in the cytoplasm and the nuclei of Huh7 cells were observed by electron microscopy (marked by arrows). Bar: $1 \mu \mathrm{m}$ or $500 \mathrm{~nm}$. (D) CD46 on the surface of Huh7 cells was detected by IHC. Bar: $100 \mu \mathrm{m}$.
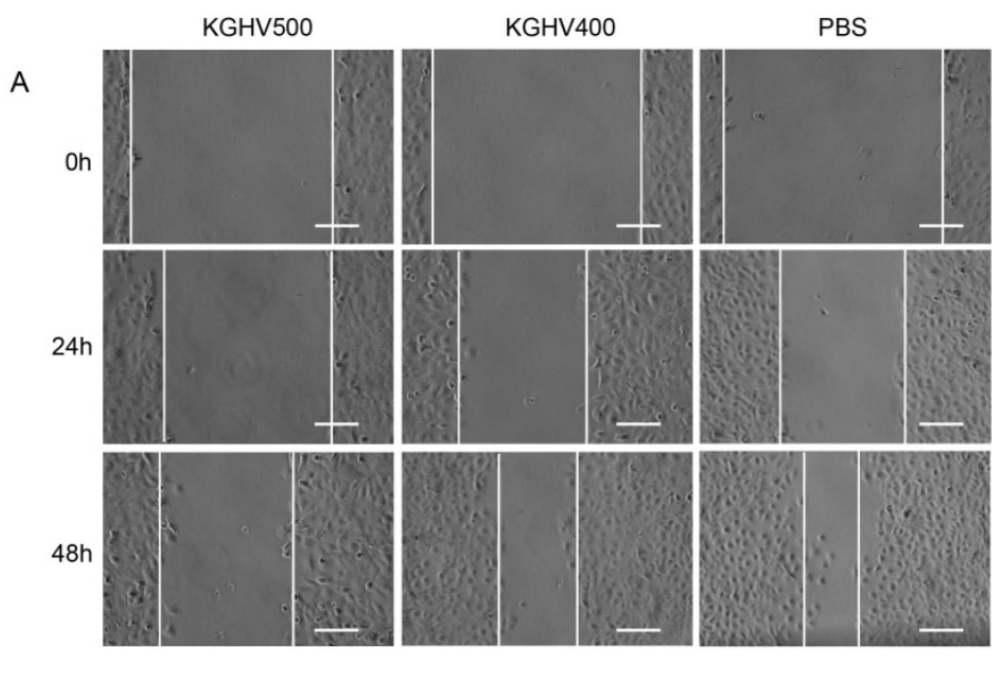

D
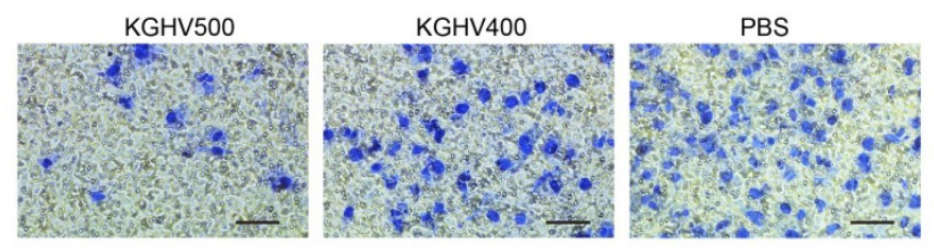

$\mathrm{F}$

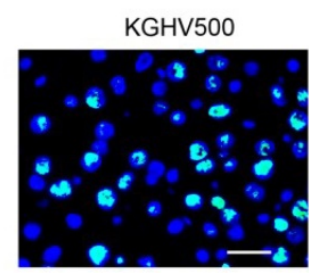

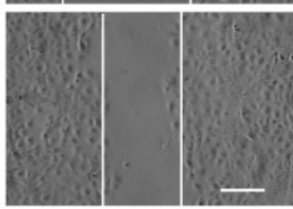

KGHV400
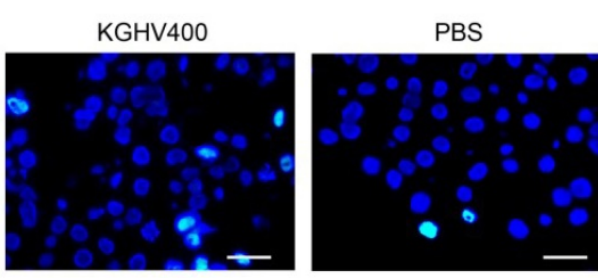
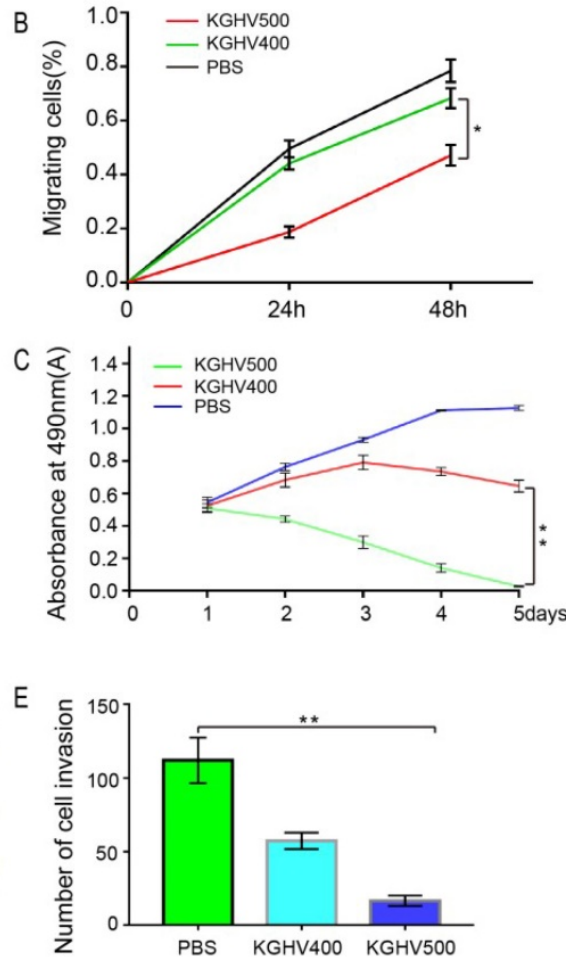

G

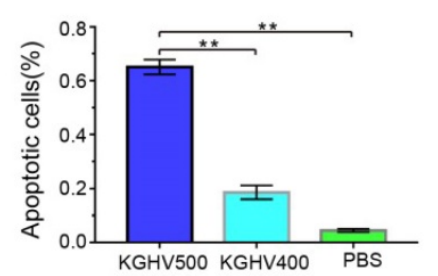

Figure 2. In vitro anti-tumor effects of KGHV500. (A and B) In wound healing assay, healing in KGHV500 group was slower than in KGHV400 or PBS group, and the number of cells that migrated was less than the other groups $(\mathrm{P}<0.05)$ Bar: $50 \mu \mathrm{m}$. (C) In MTT assay, KGHV500 was more toxic to the cells than KGHV400 or PBS (P<0.01). (D and E) In Transwell assay, KGHV500-infected Huh7 cells were significantly less invasive than the other groups $(P<0.01)$. Bar: $40 \mu m(F$ and $G)$ In TUNEL assay, the number of apoptotic Huh7 cells in KGHV500 group was higher than that of other groups $(\mathrm{P}<0.01)$. Bar: $100 \mu \mathrm{m} * \mathrm{P}<0.05, * * \mathrm{P}<0.01$. 

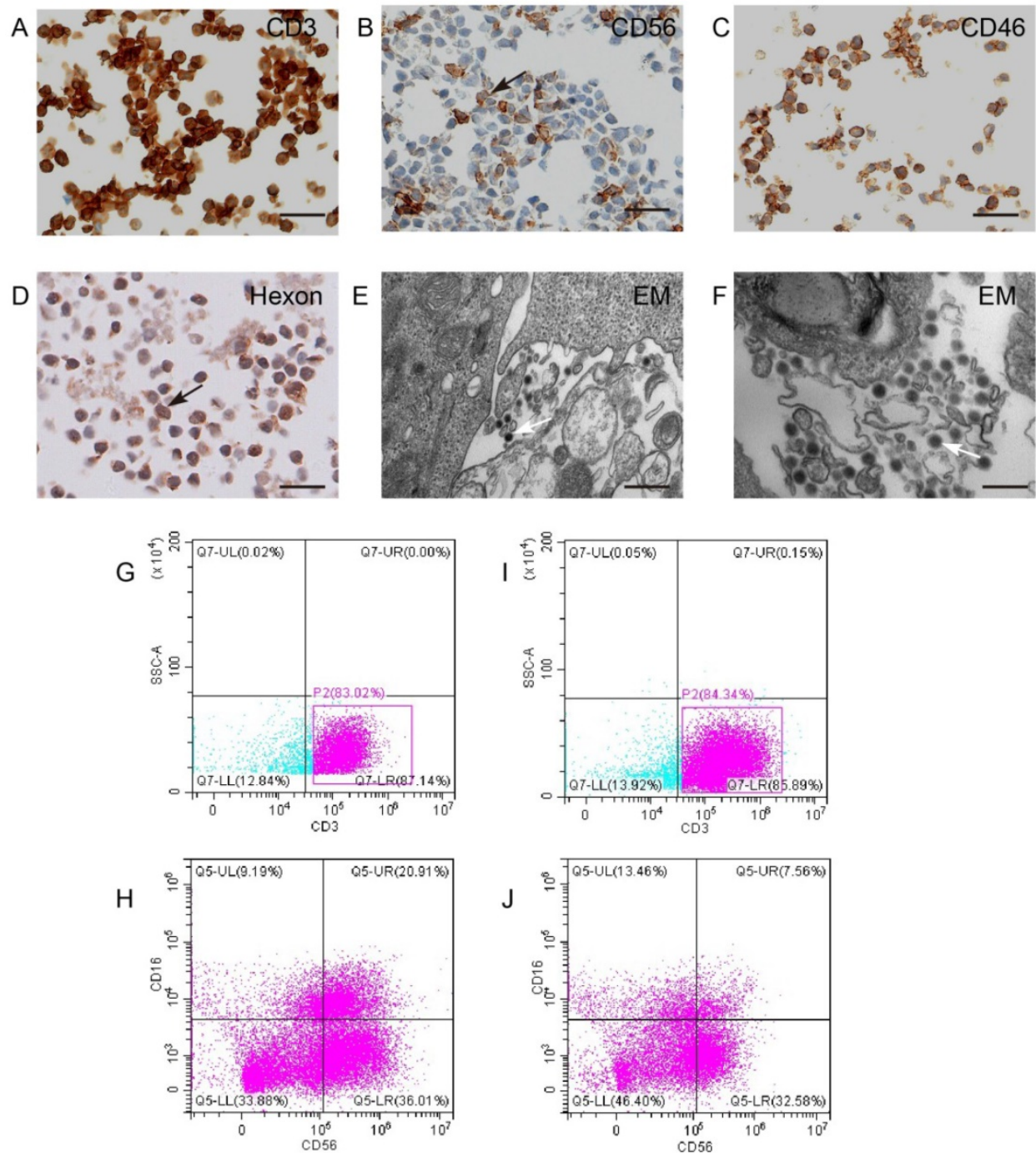

Figure 3. CIK cells could carry KGHV500. (A and B) CIK cell markers CD3 and CD56 were detected by IHC. Bar: $100 \mu$ m. (C) CD46 on the surface of CIK cells was detected by IHC. Bar: $100 \mu \mathrm{m}$. (D) The adenovirus hexon was detected in CIK cells by IHC. Bar: $100 \mu \mathrm{m}$. Strong staining was marked by arrows. (E and F) Electron microscopy showed that many virus particles accumulated on CIK cell membrane (marked by arrows). Bar: $500 \mathrm{~nm}$. (G-J) Flow cytometry of CD3 and CD56 expression of CIK cells before and after KGHV500 infection.

\section{KGHV500 inhibited liver cancer cell growth}

MTT assay showed that cell viability of KGHV500-treated group decreased over time, while cell viability of KGHV400-treated group increased initially and decreased slowly, indicating that KGHV500 inhibited Huh7 cell growth (Figure 2C).

\section{KGHV500 inhibited liver cancer cell invasion}

Transwell assay demonstrated that the number of invading cells in KGHV500-treated group was less than that in other groups (Figure 2D). The number of invading cells in KGHV500 group was $16.20 \pm 3.19$, compared to $57.40 \pm 5.00$ in KGHV400 group and $112.00 \pm 13.83$ in control group (Figure 2E).

\section{KGHV500 induced apoptosis in liver cancer cells}

TUNEL assay showed that KGHV500 treatment increased the number of apoptotic cells compared to the other groups (Figure 2F). The percentage of apoptotic cells was $65.08 \pm 2.72 \%$ in KGHV500-treated group, $18.59 \pm 2.59 \%$ in KGHV400-treated group, and $4.43 \pm 0.59 \%$ in control group (Figure $2 \mathrm{G}$ ).

\section{KGHV500 bound to CIK cells}

CIK cells were identified by surface specific markers CD3 and CD56 (Figure 3A,B). To test whether KGHV500 could infect CIK cells, immunohistochemistry was used to detect CD46 (Figure 3C) and adenovirus hexon (Figure 3D) on CIK cell surface. Electron microscopy confirmed the presence of KGHV500 particles on the surface of CIK cells (Figure 3E,F). In addition, we performed flow cytometry to detect the changes in the phenotypes of CIK cells before and after KGHV500 infection (Figure 3G-J), and found that CD3 positive cells before and after infection were greater than $80 \%$, while 
$\mathrm{CD}^{+}{ }^{+} \mathrm{CD} 16^{+} \mathrm{CD} 56^{+}$cells decreased from $20.91 \%$ before infection to $7.56 \%$ after infection. These results indicated that CIK cells were successfully infected and could bind recombinant adenovirus KGHV500.

\section{In vivo anti-tumor efficacy of CIK cells carrying KGHV500}

Tumor volume of KGHV500 + CIK cells treated mice was the smallest, indicating that KGHV500 + CIK cells significantly inhibited the growth of mouse xenograft tumors (Figure 4A). TUNEL assay indicated that the apoptotic rate of KGHV500 + CIK group was $81.50 \pm 1.72 \%$, higher than that of KGHV400 + CIK group $(55.53 \pm 2.82 \%)$, KGHV500 group (19.47 \pm $2.29 \%)$, CIK cell group (11.33 $\pm 0.93 \%)$, and PBS group $(7.27 \pm 0.77 \%)$ (Figure $4 \mathrm{~B})$. In addition, we detected the expression of apoptosis-related genes in the xenograft tissue by PCR and Western blot analysis (Figure $4 \mathrm{C}, \mathrm{D})$. KGHV500 + CIK treatment decreased the expression of anti-apoptotic proteins $\mathrm{Bcl}-2$ and survivin and increased the expression of pro-apoptotic proteins p53, caspase-3, and caspase-7. These data indicated that KGHV500 + CIK cells induced apoptosis in Huh7 liver cancer cells and inhibited tumor growth in nude mice.

\section{Safety and distribution of KGHV500 + CIK cells in vivo}

Next we performed immunohistochemistry and Western blotting to detect the distribution of adenovirus hexon and anti-p21Ras scFv in tumor and normal tissues and determine the safety of KGHV500 + CIK cells.

In KGHV500 + CIK treatment group, adenovirus hexon was expressed in tumor tissue, the spleen, liver, and kidney, but not in the lung, heart, stomach, pancreas, large intestine, small intestine, or brain tissue. In KGHV500 treatment group, adenovirus hexon was detected in both tumors and normal tissue, except for brain tissue (Figure 5A). The percentages of

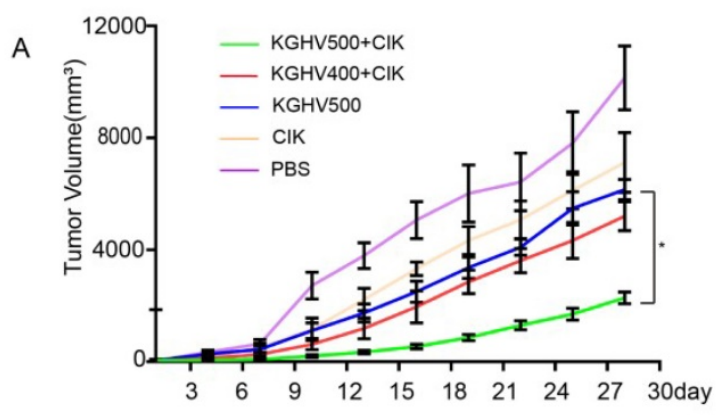

B

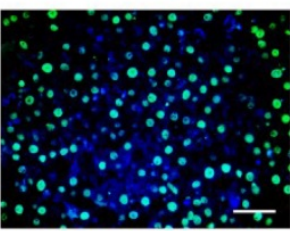

KGHV500+CIK

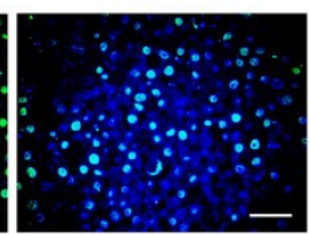

KGHV400+CIK

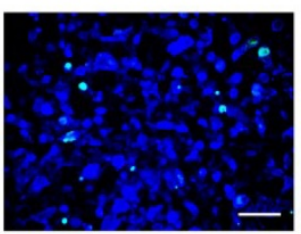

KGHV500

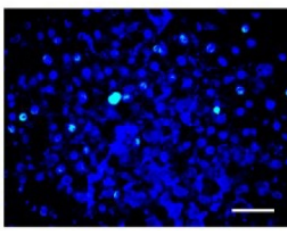

CIK

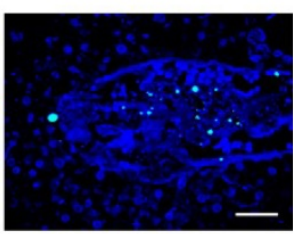

PBS
C

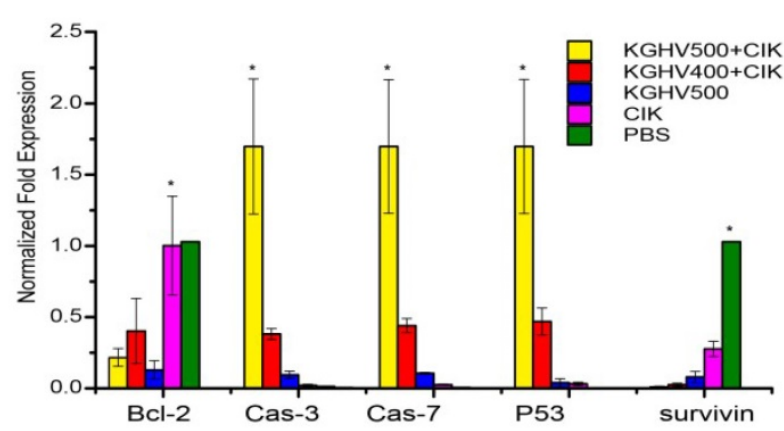

D
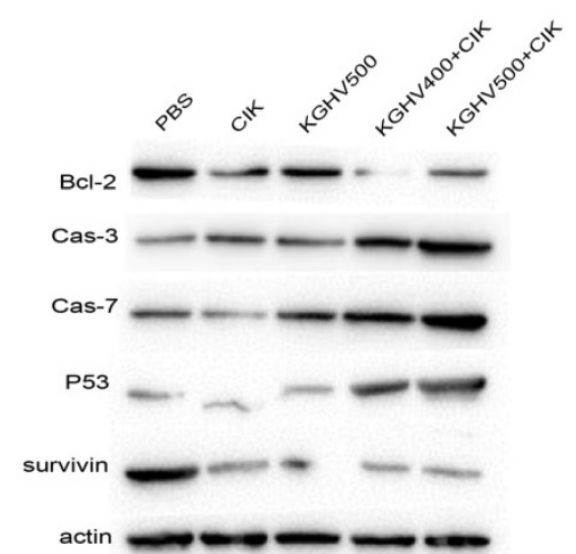

Figure 4. Anti-tumor efficacy of CIK cells carrying KGHV500 in vivo. (A) Tumor growth curve showed that CIK + KGHV500 tumor size was significantly smaller compared to other groups. (B) TUNEL assay of apoptotic tumor cells in each group. Bar: $100 \mu \mathrm{m}$. (C) The mRNA expression of apoptosis-related genes in xenografts was detected by PCR. $* P<0.05$. (D) Western blot analysis of apoptosis-related proteins in xenografts. 
adenovirus hexon positive cells in tumors from CIK + KGHV500 group were $25.45 \pm 2.48 \%, 42.67 \pm 0.72 \%$, $57.56 \pm 1.23 \%$, and $85.22 \pm 1.40 \%$ on days $1,3,5$, and 7 after administration, respectively. In KGHV500 group, the percentages were $9.89 \pm 0.42 \%, 22.33 \pm$ $0.98 \%, 34.44 \% \pm 1.40 \%$, and $49.78 \pm 1.50 \%$ on days 1,3 , 5 , and 7 after administration, respectively (Figure 5B). The percentage of adenovirus hexon positive cells in xenograft tumors was higher in CIK + KGHV500 group than in KGHV500 group. These results indicate that CIK cells carrying recombinant adenovirus have tumor targeting abilities and can deliver recombinant adenovirus to tumor site.

Immunohistochemistry demonstrated that the percentages of anti-p21Ras $\mathrm{scFv}$ positive cells in xenograft tumors from CIK + KGHV500 group were $19.67 \pm 0.98 \%, 35 \pm 1.36 \%, 53 \pm 0.98 \%$, and $75.33 \pm$ $1.79 \%$ on days $1,3,5$, and 7 after administration, respectively. In KGHV500 group, the percentages were $4.11 \pm 0.69 \%, 13.44 \pm 1.38 \%, 21.55 \pm 0.88 \%$, and $32.78 \pm 2.08 \%$, on days $1,3,5$, and 7 after administration, respectively (Figure 5C). Therefore, carrying KGHV500 in CIK cells increased the expression of anti-p21Ras scFv in xenograft tumors.
A
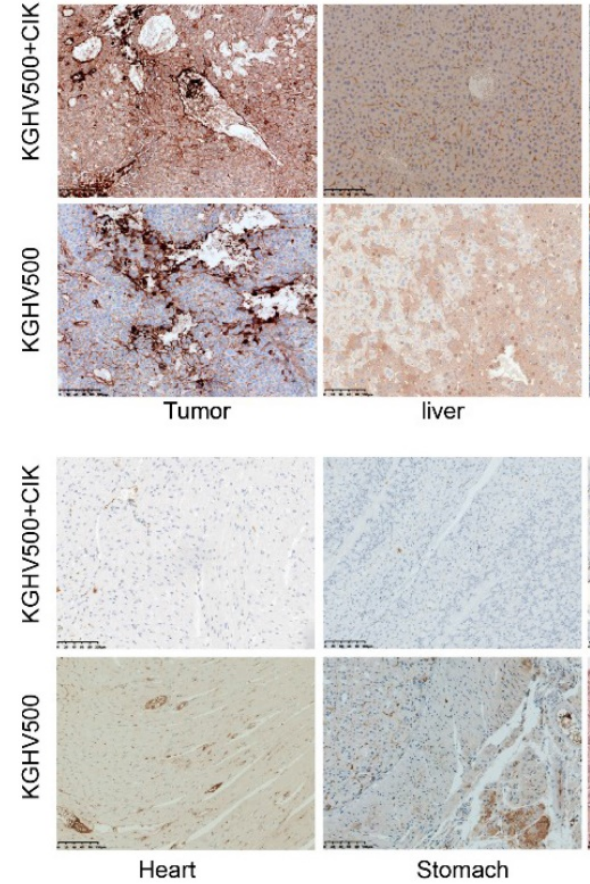

B
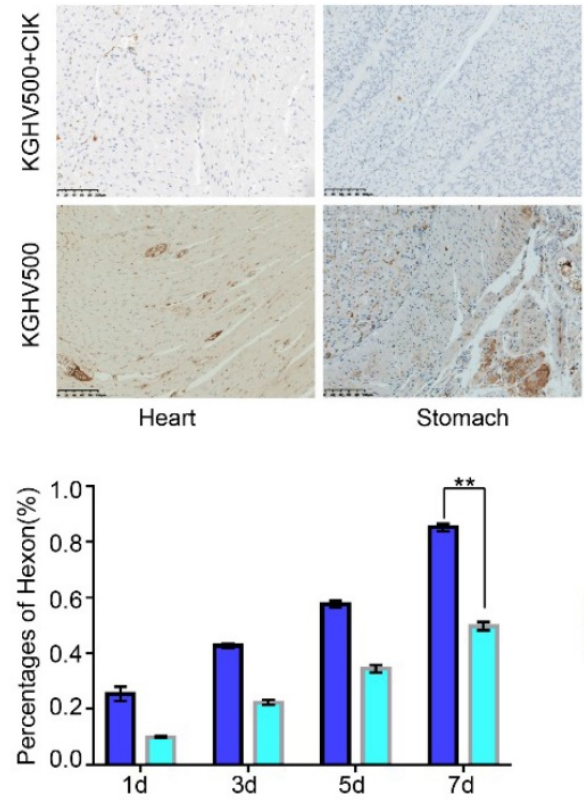
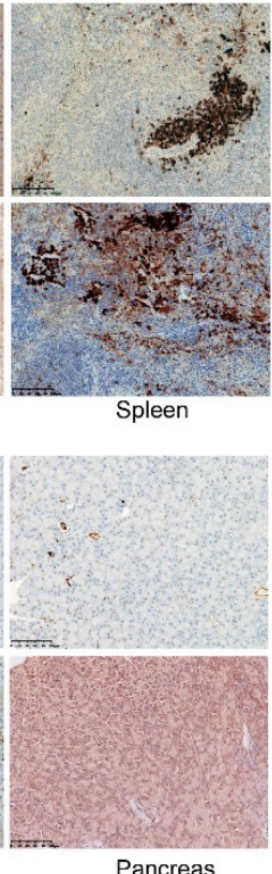

Pancreas

\begin{abstract}
C
\end{abstract}
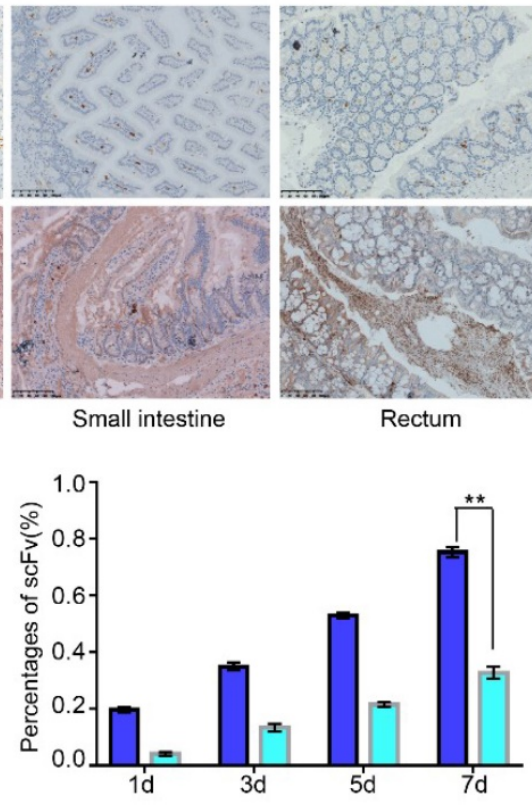

Small intestine

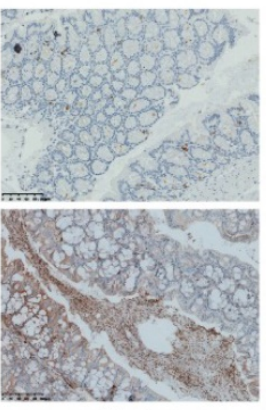

KGHV500+CIK - KGHV500

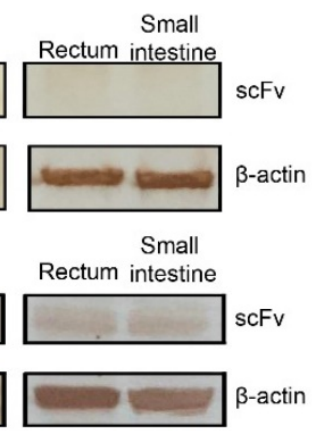

Figure 5. Targeting and safety of CIK + KGHV500 cells in vivo. (A) Expression of adenovirus hexon was detected by IHC. Bar: $100 \mu \mathrm{m}$. (B) In CIK + KGHV500 group, the percentage of tumors positive for adenovirus hexon was higher than that in KGHV500 group. **P $<0.01$. (C) CIK $+K G H V 500$ group had more anti-p21 Ras scFv-positive cells in xenograft tumors than the KGHV500 group. $* * \mathrm{P}<0.01$. (D) Western blot analysis of anti-p21 Ras scFv expression in xenograft tumors and organs of nude mice. 
Western blotting confirmed that in KGHV500 + CIK group, anti-p21 Ras scFv was expressed in tumor tissue, the liver, spleen, and kidney, while in KGHV500 group, anti-p21 Ras scFv was expressed in tumor tissue and all normal tissues, except for brain tissue (Figure 5D). Expression of anti-p21Ras scFv was consistent with the distribution of adenovirus hexon, indicating that CIK cells delivered KGHV500 to tumor tissues selectively to express anti-p21 Ras scFv.

\section{Discussion}

RAS plays an oncogenic role in cancer development [10-13]. Therefore, Ras inhibition is a potential strategy to treat liver cancer. The anti-p21Ras scFv constructed in our laboratory can block Ras signaling and target tumors with high expression of Ras $[14,15]$. However, there are hurdles to the application of anti-p21Ras $\mathrm{scFv}$ in cancer treatment, including efficient delivery to tumor cells, intracellular p21Ras protein binding, and achievement of sustained expression.

Oncolytic adenovirus continuously infects tumor tissues, allowing for efficient infection and maintenance of long-term gene expression [17]. Moreover, it does not replicate in normal tissues, but replicates in cancer cells to kill cancer cells selectively [18]. Therefore, we constructed recombinant oncolytic adenovirus KGHV500 that could specifically replicated in tumor cells and expressed anti-p21Ras scFv. In vitro experiments showed that KGHV500 successfully infected liver cancer cells, expressed antip21Ras scFv, inhibited liver cancer cell proliferation, migration, invasion, and induced apoptosis of liver cancer cells.

Although oncolytic adenovirus-mediated gene therapy has many advantages by intratumoral delivery, the delivery in vivo is a limiting factor since it could infect normal cells. For intravenous injection of virus, the virus is non-specifically adsorbed by vascular endothelial cells and blood cells, or neutralized by antibodies [19]. In order to transport KGHV500 to tumor tissues and enhance the targeting and safety in vivo, we used CIK cells to carry KGHV500 to tumor tissues.

By immunohistochemistry and flow cytometry, we confirmed that CIK cells we induced had high expression of CD3 and CD56. CIK cells specifically recognize tumors without toxic effects on normal cells. CIK cells recognize tumors mainly by chemokine ligand (CXCL) 9, CXCL10, and CXCL11, or by binding of CIK cell surface NKG2D receptor to tumor cell surface MHC class I chain-related protein A or B ligand [20,21]. Clinical studies have shown that CIK cell therapy alone is less responsive to patients with high tumor burden [22]. Therefore, combining CIK cell therapy with oncolytic adenovirus treatment may be a better approach for cancer therapy.

Furthermore, we found KGHV500 distribution and anti-p21Ras scFv expression in the liver, spleen and kidney of nude mice. Adenovirus accumulation in the liver is associated with hepatotoxicity [23]. In addition, $90 \%$ of intravenous adenoviruses are excreted by the liver [24]. Adenovirus accumulation has been detected in the spleen, which may be related to the homing of peripheral lymphocytes [25]. Detection of recombinant adenovirus in the kidney may be related to kidney metabolism [26]. Our results indicate that CIK cells carrying KGHV500 significantly inhibited tumor growth in vivo, and were safe with little effect on normal tissues. In addition, our previous studies showed that CIK carrying KGHV500 inhibited the growth of lung cancer, colon cancer and gastric cancer in nude mice, indicating broad-spectrum anti-tumor efficacy of CIK carrying KGHV500 [27-29].

In conclusion, we develop a novel strategy for the treatment of Ras-driven liver cancer by combining CIK cells with oncolytic adenovirus expressing antip21Ras scFv. Further preclinical studies are necessary to validate the potential of our strategy for the treatment of various cancers.

\section{Acknowledgements}

This study was supported by Major Science and Technology Project of the Yunnan Science and Technology Plan (2018ZF009), Applied Basic Research Project of 920th Hospital of Joint Logistics Support Force (NO 2019YGB02), and National Natural Science Foundation of China (81460464).

\section{Competing Interests}

The authors have declared that no competing interest exists.

\section{References}

1. Bray F, Ferlay J, Soerjomataram I, et al. Global cancer statistics 2018: GLOBOCAN estimates of incidence and mortality worldwide for 36 cancers in 185 countries. CA Cancer J Clin 2018;68:394-424.

2. Chen W, Zheng R, Baade PD, et al. Cancer statistics in China, 2015. CA Cancer J Clin 2016;66:115-132.

3. He JJ, Zhang LY, Guo D, et al. Sinomenium Inhibits the Viability of Hepatoma Carcinoma Cells through Activating IFNA2. Oncologie 2020; 22: 1-12.

4. Chuma M, Terashita K, Sakamoto N. New molecular targeted therapies against advanced hepatocellular carcinoma: from molecular pathogenesis to clinical trials and future directions. Hepatol Res 2015;45:E1.

5. Lee $\mathrm{YH}$, Seo D, Choi KJ, et al. Antitumor effects in hepatocarcinoma of isoform-selective inhibition of HDAC2. Cancer Res. 2016;74:4752-4761.

6. Liu L, Cao Y, Chen C, et al. Sorafenib blocks the RAF/MEK/ERK pathway, inhibits tumor angiogenesis, and induces tumor cell apoptosis in hepatocellular carcinoma model PLC/PRF/5. Cancer Res 2006;66:11851-11858.

7. Chang YS, Adnane J, Trail PA, et al. Sorafenib (BAY 43-9006) inhibits tumor growth and vascularization and induces tumor apoptosis and hypoxia in RCC xenograft models. Cancer Chem Pharmacol 2007;59:561-574.

8. Zhu AX. Beyond sorafenib: novel targeted therapies for advanced hepatocellular carcinoma. Expert Opin Investig Drugs 2010;19:663-672. 
9. Montes FQ, Vázquez-Hernández A, Fenton-Navarro B. Active compounds of medicinal plants, mechanism for antioxidant and beneficial effects. Phyton, Int J Exp Botany 2019;88:1-10.

10. Sampath P, Li J, Hou W, et al. Crosstalk Between Immune Cell and Oncolytic Vaccinia Therapy Enhances Tumor Trafficking and Antitumor Effects. Mol Therapy. 2013;21:620-628.

11. Goldfinger LE, Michael JV.: Regulation of Ras signaling and function by plasma membrane microdomains. Biosci Trends 2017;11:23-40.

12. Alberto FM, Eugenio S. Ras in cancer and developmental diseases. Genes Cancer 2011;2:344-358.

13. Zhang HL, Xing XW, Liu Y, Li SL, Li WY. Comprehensive Network Analysis of Different Subtypes of Molecular Disorders in Lung Cancer. Oncologie 2020; 22: 107-116.

14. Yang JL, Liu DX, Zhen SJ, et al. A novel anti-p21Ras scFv antibody reacting specifically with human tumour cell lines and primary tumour tissues. BMC Cancer 2016;16:131.

15. Pan XY, Liu XJ, Li J, et al. The antitumor efficacy of anti-p21Ras scFv mediated by the dual-promoter-regulated recombinant adenovirus KGHV300. Gene Therapy 2017;24:40-48.

16. Jimenez-Gonzalez M, Plaza-Garcia S, Arizeta J, et al. A longitudinal MRI study on lymph nodes histiocytosis of a xenograft cancer model. PloS one 2017;12:e181043.

17. Twumasi-Boateng K, Pettigrew JL, Kwok YYE, Bell JC, Nelson BH. Oncolytic viruses as engineering platforms for combination immunotherapy. Nat Rev Cancer 2018;18:419-432.

18. Laer DV, Muik A, Stubbert LJ, et al. Re-engineering Vesicular Stomatitis Virus to Abrogate Neurotoxicity, Circumvent Humoral Immunity, and Enhance Oncolytic Potency. Cancer Res 2014;50:S199.

19. Lesniak MS, Yang Z, Zhang Q, et al. Combined Therapy with CytokineInduced Killer Cells and Oncolytic Adenovirus Expressing IL-12 Induce Enhanced Antitumor Activity in Liver Tumor Model. PLOS ONE 2012;7:e44802.

20. Hazelrigg MR, Hirsch JI, Merchant RE. Distribution of adoptively transferred, tumor-sensitized lymphocytes in the glioma-bearing rat. J Neuro-oncol 2002; 60:143-150.

21. Thorne $\mathrm{SH}$, Negrin RS, Contag $\mathrm{CH}$. Synergistic antitumor effects of immune cell-viral biotherapy. Science 2006;311:1780-1784

22. Hontscha C, Borck Y, Zhou H, Messmer D, Schmidt-Wolf IGH. Clinical trials on CIK cells: first report of the international registry on CIK cells (IRCC). J Cancer Res Clin Oncol 2011;137:305-310.

23. Wang Z, Yu B, Wang B, et al. A novel capsid-modified oncolytic recombinant adenovirus type 5 for tumor-targeting gene therapy by intravenous route. Oncotarget 2016;7:47287-47301.

24. Kuzmin AI, Finegold MJ, Eisensmith RC. Macrophage depletion increases the safety, efficacy and persistence of adenovirus-mediated gene transfer in vivo. Gene Therapy 1997;4:309-316.

25. Hong W, Fenglin C, Jinmei L, et al. Homing of cytokine-induced killer cells during the treatment of acute promyelocytic leukemia. Int $\mathrm{J}$ Hematol 2014;100:165-170.

26. Greish K, Frandsen J, Scharff S, et al. Silk-elastinlike protein polymers improve the efficacy of adenovirus thymidine kinase enzyme prodrug therapy of head and neck tumors. J Gene Med 2010;12:572-579.

27. Lin XR, Zhou XL, Feng Q, et al. CIK cell-based delivery of recombinant adenovirus KGHV500 carrying the anti-p21Ras scFv gene enhances the anti-tumor effect and safety in lung cancer. J Cancer Res Clin Oncol 2019;145:1123-1132.

28. Liu FR, Bai S, Feng Q, et al. Anti-colorectal cancer effects of anti-p21Ras scFv delivered by the recombinant adenovirus KGHV500 and cytokine-induced killer cells. BMC Cancer. 2018;18:1087.

29. Wang M, Hong Y, Feng Q, et al. Recombinant Adenovirus KGHV500 and CIK Cells Codeliver Anti-p21-Ras scFv for the Treatment of Gastric Cancer with Wild-Type Ras Overexpression. Mol Ther Oncolytics. 2018;11:90-101. 